\title{
Les mécanismes de gouvernance des entreprises familiales : Résultats, voies de recherche et préconisations managériales
}

\author{
Thierry Poulain-Rehm \\ Professeur des Universités \\ Doyen de la Faculté de Droit, de Science politique et de Gestion de La Rochelle \\ Directeur de l'IAE La Rochelle
}

\begin{abstract}
Résumé : L'objectif de cette article est d'apporter une contribution à la connaissance des mécanismes de gouvernance tant généraux que spécifiques des entreprises familiales, en tentant d'apporter des éléments de réponses à une question centrale : quelle gouvernance pour maximiser la valeur des entreprises familiales ? La recherche de pérennité et de légitimité de l'entreprise familiale au regard de la société, de ses normes et de ses valeurs, induirait l'obligation pour l'entreprise mettre en œuvre une gouvernance susceptible de maximiser l'intérêt de ses parties prenantes et, en cela, la valeur. Pourtant, des travaux tendent à montrer, que les entreprises familiales souffriraient comparativement aux entreprises non familiales d'une gouvernance de qualité inférieure. Cela doit conduire à analyser, successivement, l'efficience de leurs mécanismes de gouvernance tant généraux que spécifiques. Cet article procède à la synthèse de quelques résultats empiriques, énonce plusieurs voies de recherche et formule plusieurs préconisations managériales.
\end{abstract}

\section{Introduction}

Quelle gouvernance pour maximiser la valeur? Définie comme l'ensemble des relations entre la direction de l'entreprise, son conseil d'administration, ses actionnaires et ses autres parties prenantes (OCDE, 2004), la «corporate governance» place au centre des préoccupations le management par la valeur. Si l'interrogation concerne l'ensemble des entreprises, elle revêt une acuité particulière dans les entreprises familiales, dans la diversité de leur réalité.

L'analyse de la gouvernance des entreprises familiales, que nous avons proposé de définir comme « une entreprise dans laquelle un groupe d'actionnaires unis par des liens familiaux possède une part significative du capital et des droits de vote et exerce une influence effective sur le pouvoir de direction (...) » (Poulain-Rehm, 2006) répond à plusieurs motivations. Celle tout d'abord de contribuer à l'édification de la connaissance sur un objet d'études encore relativement peu exploré. Le paradoxe d'une prédominance dans le tissu entrepreneurial contemporain des entreprises familiales, qui de surcroît ont constitué historiquement le socle du développement des nations occidentales, et d'une relative méconnaissance des principes de management et des niveaux de performance qui caractérisent ces entreprises, et plus encore, de leur gouvernance, ne peut qu'attiser la curiosité intellectuelle et satisfaire la quête de sens du chercheur. Celle aussi de lutter contre les préjugés, en l'occurrence ceux qui ont longtemps vu dans l'entreprise familiale une forme d'organisation désuète, dont le déclin présumé face au capitalisme managérial serait engagé de manière irréversible, et dont l'opacité supposée empêcherait toute velléité d'investigation empirique. 
Le $X^{\text {ème }}$ siècle s'est caractérisé par une mutation profonde des structures des entreprises familiales. L'accroissement de la taille des entreprises familiales et l'ouverture de leur capital rendu nécessaires par les impératifs de développement ont complexifié l'analyse en faisant évoluer le modèle familial, tant du point de vue de la structure de propriété que de l'exercice du management. Le modèle d'agence traditionnel actionnaires/dirigeants apporte un éclairage utile en vue de la compréhension du fonctionnement des entreprises familiales cotées, mais partiel et applicable sous conditions (Poulain-Rehm, 2005, 2006). Une vision approfondie des problèmes de gouvernance de ces firmes, dans leur diversité, implique d'élargir le champ d'investigation et d'intégrer, de manière complémentaire ou alternative, un certain nombre de variables clés reflétant la diversité des mécanismes de gouvernance d'entreprise, de manière générale, et la diversité des mécanismes de gouvernance qui leur sont spécifiques. Dans les entreprises familiales, en effet, la recherche de pérennité et de légitimité de l'organisation au regard de la société, de ses normes et de ses valeurs, revêt une signification particulière. Par extension, cette légitimité reflèterait l'obligation pour l'entreprise de s'aligner sur les intérêts de ses parties prenantes, parmi lesquelles les membres de la famille, qu'ils soient actionnaires ou non, dirigeants ou non, occupent une place centrale.

L'objectif de cette article est de contribuer à la connaissance des mécanismes de gouvernance tant généraux que spécifiques des entreprises familiales, en tentant d'apporter des éléments de réponses à la question séminale : quelle gouvernance pour maximiser la valeur des entreprises familiales ?

Après avoir procédé à la synthèse de quelques résultats (section 1), nous esquisserons quelques voies de recherche (section 2) et formulerons quelques préconisations managériales (section 3).

\section{Le constat}

Dans l'article que nous avons consacré à «La responsabilité sociétale des entreprises familiales : une approche internationale » (Hirigoyen et Poulain-Rehm, 2014), nous avons analysé l'impact de la nature familiale des entreprises sur leur responsabilité sociétale, dans ses différentes dimensions: ressources humaines, droits humains sur les lieux de travail, engagement sociétal, respect de l'environnement, comportement sur les marchés, mais aussi la gouvernance. Les résultats, obtenus sur un échantillon de 363 entreprises cotées et réparties sur trois zones géographiques (Amérique, Europe, Asie-Pacifique), montrent que les entreprises familiales ne se distinguent pas des entreprises non familiales pour cinq des dimensions étudiées. Il n'y a pas de différences statistiquement significatives, entre firmes familiales et firmes non familiales, pour les scores de ressources humaines, droits humains sur les lieux de travail, engagement sociétal, respect de l'environnement et comportement sur les marchés. De surcroît, les entreprises familiales présentent, pour la dernière des dimensions étudiées de la responsabilité sociétale, la gouvernance, des scores inférieurs, de manière statistiquement significative, pour chacune des sous-dimensions qui la constituent : équilibre des pouvoirs et efficacité du conseil d'administration, audit et mécanismes de contrôle, engagement avec les 
actionnaires et structure de l'actionnariat, et détermination des rémunérations des principaux dirigeants.

Tableau 1.- Scores de responsabilité sociétale

Tests $t$ de différences de moyennes Entreprises familiales/entreprises non familiales

\begin{tabular}{|l|l|l|l|}
\hline & $\begin{array}{l}\text { Entreprises } \\
\text { non familiales }\end{array}$ & $\begin{array}{l}\text { Entreprises } \\
\text { familiales }\end{array}$ & $\begin{array}{l}\text { Test t } \\
\text { Student }\end{array}$ \\
\hline CG : Gouvernance & 52.12 & 45.10 & $-3.928^{* * *}$ \\
\hline CG1 : Equilibre des pouvoirs et efficacité du CA & 50.10 & 43.45 & $-2.606^{* * *}$ \\
\hline CG2 : Audit et mécanismes de contrôle & 60.98 & 57.01 & $-2.138^{* *}$ \\
\hline $\begin{array}{l}\text { CG3 : Engagement envers les actionnaires et structure } \\
\text { de l'actionnariat }\end{array}$ & 59.57 & 51.85 & $-2.861^{* * *}$ \\
\hline $\begin{array}{l}\text { CG4 : Détermination des rémunérations des principaux } \\
\text { dirigeants }\end{array}$ & 38.75 & 28.94 & $-3.893^{* * *}$ \\
\hline $\begin{array}{l}* * * / * * * \text { Significatifs respectivement au seuil de 1\%, } \\
5 \% \text { et } 10 \%\end{array}$ & & & \\
\hline
\end{tabular}

Source : Hirigoyen et Poulain-Rehm (2014)

De même, en cohérence avec ces derniers résultats, l'étude réalisée montre que les scores de gouvernance sont sensiblement inférieurs dans les entreprises à direction familiale, de manière statistiquement significative : au seuil de $1 \%$ pour le score global de gouvernance, le score d'engagement envers les actionnaires et le score représentatif de la rémunération des dirigeants ; au seuil de 5\% pour ce qui est de la dimension « audit et mécanismes de contrôle ». La dimension « équilibre des pouvoirs et efficacité du conseil d'administration » ne présente toutefois plus de différence significative.

Tableau 2.- Scores de gouvernance

Entreprises à direction familialelentreprise à direction non familiale

\begin{tabular}{|l|l|l|l|}
\hline & $\begin{array}{l}\text { Entreprises à } \\
\text { direction non } \\
\text { familiale }\end{array}$ & $\begin{array}{l}\text { Entreprises à } \\
\text { direction } \\
\text { familiale }\end{array}$ & $\begin{array}{l}\text { Test t } \\
\text { Student }\end{array}$ \\
\hline CG : Gouvernance & 51.75 & 43.74 & $3.691^{* * *}$ \\
\hline CG1 : Equilibre des pouvoirs et efficacité du CA & 49.46 & 44.38 & 1.631 \\
\hline CG2 : Audit et mécanismes de contrôle & 60.77 & 56.31 & $1.978^{* *}$ \\
\hline $\begin{array}{l}\text { CG3 : Engagement envers les actionnaires et structure } \\
\text { de l'actionnariat }\end{array}$ & 59.55 & 47.48 & $3.714^{* * *}$ \\
\hline $\begin{array}{l}\text { CG4 : Détermination des rémunérations des principaux } \\
\text { dirigeants }\end{array}$ & 38.29 & 26.64 & $3.806^{* * *}$ \\
\hline $\begin{array}{l}* * * / * * / * \text { Significatifs respectivement au seuil de 1\%, } \\
5 \% \text { et } 10 \%\end{array}$ & & & \\
\hline
\end{tabular}

Source : Hirigoyen et Poulain-Rehm (2014)

Qu'ils soient obtenus sur l'échantillon des entreprises familiales ou des entreprises à dircetion familiale, les résultats mettent en lumière des faiblesses dans l'équilibre des pouvoirs et l'efficacité du conseil d'administration, des faiblesses en matière d'audit et de mécanismes 
de contrôle, des faiblesses dans l'engagement envers les actionnaires, notamment minoritaires, et des faiblesses dans la détermination de la rémunération des dirigeants.

Plusieurs séries de facteurs peuvent expliquer ces insuffisances (Poulain-Rehm et Hirigoyen, 2014).

La première tient à la fonction d'utilité de l'actionnaire familial qui, comme nous le rappelons dans l'article sus-cité, est complexe, car elle le conduit à opérer un arbitrage entre sa consommation privée, la valorisation de son patrimoine ou celui de sa famille, et la valorisation du patrimoine de son entreprise (Mahérault, 1996), ses revenus pécuniaires sur sa durée de vie et le bien-être de la génération suivante (Bhattacharya et Ravikumar, 1999). Basco et Perez Rodriguez (2011) soulignent la diversité des entreprises familiales et établissent une typologie distinguant trois catégories principales d'entreprises familiales en fonction des orientations retenues : les entreprises « orientées famille », qui privilégient les principes et les valeurs de la famille dans leur prise de décision, afin de répondre en priorité aux besoins et objectifs de la famille; les entreprises " orientées affaires", au sein desquelles les décisions stratégiques répondent prioritairement aux objectifs de l'entreprise et de ses besoins; les entreprises « orientées famille-affaires », qui fixent les priorités en trouvant l'équilibre entre les besoins de la famille et ceux de l'entreprise (Basco et Perez Rodriguez, 2011). Cette grille de lecture peut expliquer que les intérêts de la famille et ceux de l'entreprise l'emportent sur les intérêts des différentes parties prenantes non familiales, et ce d'autant plus que l'actionnaire familial court un important risque personnel relatif aux décisions de gestion et aux conséquences qu'elles peuvent avoir sur son patrimoine personnel et celui de sa famille. Si l'actionnaire familial est ouvert au risque à titre personnel, il l'est moins au niveau du risque global de l'entreprise dans la mesure où il a une faible capacité de diversification de son portefeuille d'actifs personnels (Xiao et al., 2001).

La deuxième série de facteurs tient à l'existence de coûts d'agence liés à des biais comportementaux au sein des entreprises familiales (Chami, 2001 ; Schulze et al., 2003). Dans la lignée des travaux de Habbershon et al. (2003), Hirigoyen (2009) propose de modifier le schéma des trois cercles de Gersick et al. (1997), qui suppose un comportement homogène et monolithique des acteurs impliqués dans l'entreprise familiale. Il suggère d'ajouter un quatrième cercle, « individu », qui tient compte explicitement des préférences des individus et de leur évolution au fil du temps, susceptibles de donner naissance à des biais comportementaux. Ces biais sont cognitifs et émotionnels, tout comme individuels et collectifs. Les différences d'attentes entre les parties prenantes extérieures à la famille et la famille pouvant créer des coûts d'agence cognitifs. L'altruisme, en particulier, est susceptible d'altérer les perceptions des dirigeants et de réduire leur capacité à surveiller et à discipliner efficacement les membres de la famille, aux dépens des autres parties prenantes, internes et externes, de l'entreprise. Il devient nécessaire d'étudier et d'analyser le capital symbolique de l'actionnaire familial et la manière dont celui-ci permet de construire un avantage compétitif spécifique, et recommandent en particulier d'affiner la notion d'implication de l'actionnariat familial par la prise en compte de l'altruisme, la stratégie de croissance et les styles de leadership (O'Boyle et al., 2012). 
Ces biais peuvent avoir, de toute évidence, un impact sur les différentes dimensions constitutives de la gouvernance d'entreprise (Hirigoyen et Poulain-Rehm, 2014).

Sur les modes de détermination de la rémunération des dirigeants tout d'abord qui, à l'instar du recrutement des autres membres de la famille dans l'entreprise, de l'évaluation de leur performance ou de leur progression de carrière, n'obéit pas nécessairement à des considérations guidées par la rationalité économique. La satisfaction des intérêts de la famille prévaudrait sur ceux des autres parties prenantes, en matière de rémunération comme dans d'autres domaines, de la même façon que prévaudraient les droits des actionnaires familiaux majoritaires sur ceux des actionnaires minoritaires.

Le constat d'une efficacité et d'une efficience plus faibles du conseil d'administration et des mécanismes d'audit et de contrôle peut aussi s'expliquer à la lumière de ces considérations. Instance privilégiée de gouvernance d'entreprise, le conseil d'administration des entreprises familiales est composé, pour une part, d'administrateurs issus de la famille, et pour une autre part, qui va dépendre de la composition du capital et de la structure d'actionnariat, d'administrateurs représentant les autres catégories d'actionnaires parmi lesquels d'autres entreprises, des banques, établissements financiers, fonds d'investissement et fonds de pension qui détiendront des parts dans le capital dans un but davantage financier que stratégique. De sorte que la plupart des décisions seront fortement influencées par des administrateurs familiaux qui veilleront, avant tout, à préserver voire renforcer le contrôle familial dans le capital et le management : face au pouvoir familial, le ou les contre-pouvoirs se trouveront ainsi fortement atténués. Et ce d'autant qu'il n'est pas rare de voir, au sein de ces entreprises, des personnes, propriétaires du capital, exercer simultanément des fonctions de direction et d'administrateurs, ce qui atténue d'autant l'impact de la gouvernance. Ajoutons à cela que l'extension de la famille, au fil de l'évolution de l'entreprise familiale et des générations qui vont l'animer, va complexifier la gouvernance. La famille va perdre de son unité et de son homogénéité. Des intérêts divergents et des dissensions se manifesteront. Des conflits apparaîtront dans la prise de décision stratégique et financière, la politique de dividendes en étant l'une des illustrations les plus fréquentes, entre les actionnaires familiaux dirigeants qui privilégieront le réinvestissement des bénéfices dans l'entreprise et les actionnaires familiaux non dirigeants et minoritaires qui revendiqueront une distribution leur assurant une rente. Conflits qui s'exprimeront au sein du conseil d'administration, auront un impact négatif sur son fonctionnement et viendront diminuer en conséquence son efficience.

Ces différents constats et facteurs d'explications soulignent la nécessité d'approfondir la connaissance des mécanismes de gouvernance des entreprises familiales.

\section{Voies de recherche futures}

Le constat d'une gouvernance de qualité inférieure doit conduire à analyser, en premier lieu, l'efficience des mécanismes généraux de gouvernance de ces entreprises, en particulier de leur conseil d'administration, instance privilégiée de gouvernance dont le rôle doit être mis en 
perspective avec leur mode de direction. Il doit conduire à étudier, en second lieu, leurs mécanismes de gouvernance spécifiques, qui intègrent la singularité de ce type d'entreprises à l'interface de deux systèmes au fonctionnement différent, la famille et l'entreprise.

\subsection{Comment renforcer l'efficience des mécanismes généraux de gouvernance de l'entreprise familiale?}

Considéré comme l'organe de contrôle le plus important dans la typologie des mécanismes de gouvernance (Fama et Jensen, 1983), le conseil d'administration a fait l'objet de nombreuses études dans le contexte général de l'entreprise managériale, aux Etats-Unis et en France. Plus rares, en revanche, ont été celles consacrées spécifiquement aux entreprises familiales, alors que le rôle disciplinaire du conseil est d'autant plus important que le capital est dominé par la famille (Charreaux et Pitol-Belin, 1990), puisque, dans ce cas de figure, le contrôle risque d'être de faible intensité en raison de la confusion propriété-décision.

L'analyse de l'efficience de la gouvernance exercée par le conseil d'administration des entreprises familiales et des interactions entre les actionnaires, les administrateurs et les dirigeants, implique d'étudier le rôle du conseil d'administration dans la détermination des orientations des activités des sociétés familiales et le contrôle de leur mise en œuvre, en s'intéressant notamment à quatre de ses caractéristiques : le cumul des fonctions de direction générale et de présidence du conseil d'administration, la qualité des administrateurs, la taille du conseil et l'existence de comités spécialisés.

Si les approches disciplinaires apportent un éclairage utile en soulignant notamment, dans le cas général, l'intérêt d'une participation au conseil d'administrateurs indépendants pour veiller aux intérêts des actionnaires, ou les risques d'une taille élevée qui favoriserait la domination des dirigeants et la formation de coalitions, une ouverture en direction des modèles cognitif et comportemental (Charreaux, 2002) de la gouvernance doit être, de manière complémentaire, opérée. Le courant cognitif, en accordant une importance majeure à la connaissance et aux compétences, ainsi qu'aux capacités des firmes à innover, offre une meilleure compréhension du processus productif de création de valeur. L'amélioration de la coordination qualitative et l'invention de nouvelles opportunités productives y sont vues comme des facteurs clés de performance. Le courant comportemental, pour sa part, permet de comprendre que l'efficacité des leviers disciplinaires est tributaire des biais comportementaux qui affectent les décisions managériales, biais qui peuvent être à l'origine de pertes de valeur plus importantes que celles liées à l'opportunisme (Charreaux, 2013). L'importance des facteurs cognitifs et comportementaux dans les entreprises familiales (Hirigoyen, 2008) souligne l'intérêt d'une analyse de l'instance privilégié de gouvernance à la lumière de ces différents prismes conceptuels.

Une recherche exploratoire, s'appuyant notamment sur l'analyse de données objectives, permettrait de mettre en exergue certaines caractéristiques-clés du conseil d'administration des sociétés familiales cotées. Cette analyse ayant vocation à être complétée par l'examen des déclarations des présidents de conseil d'administration, qui rendent notamment compte, dans 
les sociétés dont les titres financiers sont admis aux négociations sur un marché réglementé, de la composition, des conditions de préparation et d'organisation des travaux du conseil (article L. 225-37 du Code de commerce). Cette étude exploratoire pourrait également s'appuyer sur des entretiens semi-directifs avec des dirigeants et des administrateurs, familiaux et non familiaux, afin d'explorer les attitudes et de comprendre les comportements (Evrard et alii, 2003), et percevoir leur vision de la gouvernance. Une étude à caractère hypothético-déductif s'appuyant sur les enseignements tirés de la phase exploratoire permettrait de soumettre à l'épreuve des faits un modèle en mettant en relation, grâce à des analyses de régression, la composition de la structure d'actionnariat, en particulier le pourcentage de capital détenu par la famille, en relation avec le pourcentage de capital détenu par les investisseurs institutionnels et les actionnaires minoritaires, les caractéristiques des conseils d'administration (dissociation ou unicité des fonctions de président du conseil et de directeur général, pourcentage d'administrateurs non familiaux, taille du conseil, existence de comités spécialisés) et la création de valeur actionnariale (valeur ajoutée boursière et création de valeur anticipée).

Ces pistes de recherche, qui devraient être conduites dans l'idéal à la fois sur des échantillons d'entreprises familiales et non familiales, en France et à l'international, devraient permettre de dégager un certain nombre de conclusions présentant un réel intérêt managérial, quant aux conditions à réunir afin que le conseil d'administration des entreprises familiales soit mieux organisé, plus efficace et efficient, et contribue davantage à l'objectif de création de valeur.

Au-delà de la valeur pour l'actionnaire, et des mécanismes de gouvernance de nature à discipliner l'action des dirigeants dans cette direction, une appréhension de la valeur plurale de l'entreprise familiale implique d'analyser, de manière complémentaire, ses mécanismes de gouvernance spécifiques.

\subsection{Comment améliorer l'efficience des mécanismes spécifiques de gouvernance de l'entreprise} familiale?

L'entreprise familiale est singulière à bien des égards, car elle se situe à l'interface de deux systèmes au fonctionnement différent, la famille et l'entreprise. Le premier dominé par l'émotion, l'affect, la tradition et la sécurité ; le second par la rationalité, la recherche de profit, la croissance, la compétitivité et le changement (Goetschin, 1988). Compte tenu de la singularité de l'entreprise familiale, différentes problématiques spécifiques de gouvernance sont susceptibles d'émerger. Un contrôle excessif, fort, dominant, du dirigeant et de sa descendance, peut les inciter, tantôt à neutraliser les différents mécanismes disciplinaires de gouvernance dans une logique d'enracinement, tantôt à retirer des bénéfices privés de l'entreprise, c'est-à-dire des avantages financiers non proportionnels à leur participation au capital (La Porta et alii, 2000). Le lien affectif entre l'entreprise et son dirigeant serait ainsi susceptible d'exercer une influence négative sur la qualité de la prise de décision, les valeurs véhiculées dans les entreprises familiales tendant alors à affaiblir le management au détriment des intérêts des autres parties prenantes, en particulier les salariés. 
Au regard de ces singularités, l'ambition devrait être d'analyser, sujet faiblement exploré dans la littérature, l'efficience des mécanismes spécifiques de gouvernance de l'entreprise familiale, au croisement de la gouvernance de l'entreprise et de la gouvernance de la famille : le conseil de famille et la charte familiale.

Le conseil de famille, élu par les membres de la famille, traite de sujets importants entre la famille actionnaire et les dirigeants, qui concernent la stratégie à moyen et long terme, et les décisions de court terme, pour lesquels il y a risque de divergence d'opinion. Ce conseil présente ainsi une grande utilité pour le maintien de la cohésion familiale et l'évitement des conflits, au sens où il permet de confronter la position de la famille avec celle des autres administrateurs et des dirigeants. L'efficience de cette instance de gouvernance soulève un certain nombre d'interrogations, relatives notamment à sa composition : quelle représentation pour les différentes branches de la famille ? Comment assurer la prise en compte du critère de compétence dans la désignation des représentants? Quel mode opératoire retenir afin de prévenir ou surmonter les conflits? Les choix peuvent sembler évident lorsque la famille est peu nombreuse, mais s'avérer plus complexes au fil du développement de l'entreprise familiale et des générations qui se succèdent. Autre mécanisme de gouvernance spécifique : la charte familiale, document n'ayant pas de valeur juridique, ni de caractère obligatoire, mais qui formalise les valeurs et les grands principes de fonctionnement de la famille, tout en servant de guide pour l'avenir avec pour objectifs de prévenir les conflits potentiels susceptibles de porter préjudice au bon fonctionnement de l'entreprise, fixer les futures règles dans les relations famille/entreprise et proposer des solutions en cas de difficulté. L'efficience de ce mécanisme de gouvernance dépend de la nature des principes retenus et de son mode d'élaboration : quels modes de consultation de la famille ? Comment parvenir au consensus ? Quels principes édicter afin de prévenir et résoudre les conflits potentiels susceptibles de compromettre l'objectif de création de valeur pour les parties prenantes et la pérennité de l'entreprise ?

La mobilisation des approches cognitives et comportementales de la gouvernance trouverait pleinement son sens dans cette perspective. Sur un plan cognitif en effet, la gouvernance a non seulement pour mission de juguler les conflits d'intérêt au sein de l'organisation, mais aussi d'arbitrer les conflits cognitifs, en particulier ceux de nature axiologique, relatifs aux valeurs, à l'équité et à l'éthique des différentes parties prenantes de l'entreprise, afin de partager la rente organisationnelle la plus élevée et la plus durable possible entre les différentes parties prenantes. Sur le plan comportemental, l'enjeu est de juguler les erreurs comportementales (Greenfich, 2005), et les coûts induits qui viennent diminuer la création de valeur.

La réalisation d'études de cas devrait permettre d'apporter des éléments de réponse aux questionnements sus-énoncés. Ce type de méthodologie a pour objet de favoriser l'observation et la compréhension des phénomènes complexes et processuels (Wacheux, 1996), et permet la combinaison de méthodes susceptibles d'améliorer la compréhension de l'existant des acteurs, en élaborant un modèle de fonctionnement du système étudié (David, 2003). Son intérêt réside dans sa capacité à rendre compte du caractère évolutif et complexe des phénomènes concernant un système social aux dynamiques propres (Mucchielli, 2002). Le caractère particulier de la présence de la famille et de son influence dans les prises de décisions devrait conduire à 
envisager la réalisation d'entretiens, préalable nécessaire, dans une logique abductive, à une modélisation ayant vocation à être testée à plus grande échelle. Cette étape permettrait de spécifier les relations entre les variables étudiées, de déceler le contexte des prises de décisions et d'évaluer les rapports de force entre les membres de la famille, les dirigeants et les actionnaires. Les entretiens permettraient d'accéder directement aux motivations des acteurs afin de comprendre le fondement de leurs décisions et de déterminer les facteurs clés de la gouvernance. Cette méthode permettrait enfin d'évaluer la légitimité interne et externe des acteurs impliqués dans le processus. La variété des entretiens porterait sur les critères théoriques repérés comme ayant une influence sur la gouvernance : l'âge des sociétés, le nombre de membres de la famille impliqués, le capital détenu par la famille et sa concentration en mains familiales, l'appartenance du dirigeant à la famille. Ces entretiens devraient ainsi permettre d'analyser l'articulation des mécanismes de gouvernance spécifique et d'analyser le mode d'association des autres parties prenantes à la gouvernance de l'entreprise.

Au-delà de l'intérêt qu'il présenterait en termes d'enrichissement de la connaissance de la gouvernance du mode d'actionnariat patrimonial, et des enjeux et difficultés qui lui sont inhérents, les recherches à venir devraient permettre d'établir, sur le plan managérial, les liens entre gouvernance de l'entreprise et gouvernance de la famille, et d'en évaluer les conséquences sur la pérennité et la valeur des entreprises familiales.

A plus court terme, un certain nombre de préconisations managériales s'impose afin que la gouvernance des entreprises familiales, en particulier celles qui font appel public à l'épargne, gagne en efficience.

\section{Préconisations managériales}

Loin d'avoir une vocation seulement explicative, les Sciences de gestion visent à produire des connaissances et des outils de nature à faciliter la décision et l'action collective pour améliorer la performance des entreprises et, plus largement, des organisations. Elles sont, en ce sens, Sciences de l'action, Leur finalité dépasse ainsi l'ambition théorique, elle se veut pratique par l'édiction et la formulation de recommandations, de prescriptions ou de normes pour les entreprises et leurs dirigeants.

L'un des enjeux pour les entreprises familiales, comme pour les autres, est, sans conteste, de se doter de conseils dont les membres ont l'expertise et l'indépendance nécessaires pour agir dans le meilleur des intérêts des entreprises : définir la stratégie et les orientations générales, exercer un réel contrôle sur le management par des mécanismes appropriés et veiller à la protection des intérêts des actionnaires minoritaires. A côté de ces fonctions traditionnelles, les conseils doivent également assurer, comme les y incitent les Principes de gouvernance d'entreprise du G20 et de l'OCDE (2015), la surveillance des principales acquisitions et cessions d'actifs réalisées par l'entreprise et l'intégrité des systèmes de comptabilité et de communication financière, ou encore la surveillance de la gestion des risques, la planification fiscale et le contrôle interne. 
Les sociétés familiales doivent, au même titre que les autres catégories d'entreprises, également veiller à améliorer la qualité de la politique de rémunération des dirigeants, qui n'obéit pas toujours à des considérations guidées par la rationalité économique. L'objectif d'incitation à la performance doit être réaffirmé et le lien avec la performance garanti. Une transparence renforcée devrait jouer un rôle positif à cet égard : les Principes de gouvernance $d u$ G20 et de l'OCDE (2015) invitent ainsi les assemblées générales annuelles des actionnaires à se prononcer sur la rémunération des administrateurs et/ou des principaux dirigeants, et notamment sur la composante en actions de la rémunération des administrateurs, des principaux dirigeants et des salariés, les actionnaires étant directement intéressés par la manière dont les rémunérations sont liées aux résultats de la société lorsqu'ils évaluent les compétences du conseil d'administration.

Par ailleurs, les entreprises familiales faisant appel public à l'épargne doivent aussi prêter une attention particulière en matière d'engagement envers les actionnaires. Comme l'énonce l'OCDE (2015), les droits élémentaires des actionnaires doivent comprendre le droit de « bénéficier de méthodes fiables d'enregistrement de leurs titres », de " pouvoir céder ou de transférer des actions », "d'obtenir en temps opportun et de façon régulière des informations pertinentes et significatives sur la société », de « participer et de voter aux assemblées générales des actionnaires », « d'élire et de révoquer les administrateurs » et « d'être associés au partage des bénéfices de la société ». Il doit, en outre, être obligatoire de « rendre publics les structures du capital et les dispositifs de contrôle » et le fonctionnement efficace et transparent des marchés du contrôle des sociétés doit être garanti (OCDE, 2015).

Un autre point d'amélioration concerne les mécanismes d'audit et de contrôle, et notamment l'indépendance des auditeurs et leur responsabilité vis-à-vis des actionnaires, dans les entreprises managériales mais aussi dans les entreprises familiales. L'OCDE (2015) rappelle à cet égard les Principles of Auditor Independence and the Role of Corporate Governance in Auditor's Independence de l'OICV (Organisation Internationale des Commissions de Valeurs), qui précisent que « les normes d'indépendance des auditeurs doivent définir un corps de principes, conforté par un ensemble d'interdictions, de restrictions, d'autres mesures ou procédures ainsi que d'obligations de publicité, qui traite à tout le moins des menaces suivantes pesant sur cette indépendance : l'intérêt personnel, l'autocontrôle, la défense d'une cause particulière, les liens de familiarité et l'intimidation » .

Autant de principes et de recommandations de portée générale, mais qui revêtent une acuité particulière dans les sociétés familiales, en particulier celles faisant appel public à l'épargne.

\section{Conclusion}

Singulière dans son identité et sa culture, dans sa propriété comme dans ses modes de direction, les entreprises familiales le sont aussi par la pluralité et la richesse de leurs profils, ce qui constitue assurément une force et un gage de pérennité dans le tissu entrepreneurial contemporain. Dans les firmes familiales, la recherche de pérennité et de légitimité de l'organisation au regard de la société, de ses normes et de ses valeurs, revêt une signification 
particulière et conduit à penser que ce type d'entreprises promeut une gouvernance soucieuse de maximiser la valeur pour les différentes parties prenantes qui les composent. Néanmoins, l'évaluation de la qualité de leur gouvernance doit être nuancée (Hirigoyen et Poulain, Rehm, 2014). Sur le plan managérial et opérationnel, cela doit constituer pour les entreprises familiales une incitation forte à renforcer leurs efforts dans le domaine de la gouvernance. Sur le plan scientifique, cela doit inciter les chercheurs à poursuivre et prolonger leurs travaux autour des problématiques qui animent ces entreprises : la croissance et le développement, la structuration de l'actionnariat, l'internationalisation, la transmission, mais aussi la gouvernance, dans le cadre d'une approche globale et pluridisciplinaire, stratégique. juridique, fiscale, financière et humaine. 


\section{Bibliographie}

Basco R. et Perez Rodriguez M.J. (2011), « Ideal Types of Family Business Management: Horizontal Fit between Family and Business Decisions and the Relationship with Family Business Performance », Journal of Family Business Strategy, vol. 2, p. 151-165.

Bhattacharya U. et Ravikumar B. (1999), Capital Markets and Evolution of Family Businesses; Working ispepper Series; Indiana University, Kelley School of Business. Indianapolis, IN, USA, 1999.

Bebchuk L.A. et Weisbach M.S. (2010), «The State of Corporate Governance Research », Review of Financial Studies, Society for Financial Studies, vol. 23, n³, p. 939-961.

Berle A.A. et Means G.C. (1932), The Modern Corporation and Private Property. New York: Commerce Clearing House.

Chami R., What's Different About Family Businesses ?, IMF Working Paper IMF WP 01/70, 2001, http://www.imf.org/external/pubs/ft/wp/2001/wp0170.pdf.

Charreaux G. (2013), « Le gouvernement d'entreprise », in J. Allouche (coord.), Encyclopédie des ressources humaines, p. 725-732.

Charreaux G. (2002), «Variation sur le thème A la recherche de nouvelles fondations pour la finance et la gouvernance d'entreprise », Finance Contrôle Stratégie, vol. 5, n³, p. 5-68.

Charreaux G. et Pitol-Belin J.P. (1990), Le conseil d'administration, Vuibert Gestion.

Cochran P. et Wartick S. (1988). Corporate Governance: a Review of the Literature, Financial Executives Research Foundation, Morristown, New Jersey.

David A. (2003), «Etudes de cas : généralisation scientifique en sciences de gestion », Revue Sciences de Gestion, n³9, p. 139-166.

Evrard Y., Pras B. et Roux E. (2003), Market : Etudes et recherches en marketing, 3ème Edition, Paris, Dunod.

Fama E.F. et Jensen M.C. (1983), «Separation of Ownership and Control », Journal of Law and Economics, vol. 26, n², p. 301-325.

Gersick K, Davis J., Mc Collom Hampton M. et Landsberg I., Generation to Generation . Life Cycles of the Family Business, Boston, Harvard Business School Press, 1997.

Goergen M. (2007). « What do we Know about Different Systems of Corporate Governance? », Journal of Corporate Law Studies, vol. 8, $\mathrm{n}^{\circ} 1, \mathrm{p} .1-15$.

Goetschin P. (1988), « La gestion de la succession dans les PME », Revue Économique et Sociale, p. 98-107.

Greenfich (2005), «Behavioral Finance Definitions: Main Concepts », http ://perso.wanadoo.fr/greenwich/bfdef.htm.

Habbershon T.G., Williams M. et MacMillan I.C., « A unified systems perspective of family firm performance », Journal of Business Venturing (Vol. 18, 2003), p. 451-465.

Hirigoyen, G. (2008), « Biais comportementaux dans l'entreprise familiale : antécédents et impacts », Economies et Sociétés, Série « Economie de l'entreprise », K, n¹9, p. 1901-1930.

Hirigoyen, G. Concilier finance et management dans les entreprises familiales. Revue Française de Gestion 2009, 35, 393-411. (in French) istẹp.

G. Hirigoyen, T. Poulain-Rehm (2014), «La responsabilité sociétale des entreprises familiales : une approche internationale », in G. Hirigoyen, " La performance des entreprises familiales », Paris, Economica. 
La Porta R., Lopez-de-Silanes F., Shleifer A. et Vishny R. (2000), « Investor Protection and Corporate Governance », Journal of Financial Economics, vol. 58, n1-2, p. 3-27.

Mahérault, L. Comportement financier des entreprises familiales: Approche empirique. AFFI, Journée du 6 décembre 1996.

Mucchielli A. (2002), Dictionnaire des méthodes qualitatives en sciences humaines et sociales, Paris, Armand Colin.

O’Boyle E., Pollack J.M. et Rutherford M. (2012), « Exploring the Relation between Family Involvment and Firms' Performance: A Meta-Analysis of Main and Moderator Effects ", Journal of Business Venturing, p. 1-18.

OCDE (2004), Principes de gouvernement d'entreprise, http://www.oecd.org/dataoecd/32/19/31652074.PDF.

OCDE (2015). Principes de gouvernance d'entreprise du G20 et de l'OCDE, Rapport de l'OCDE aux ministres des Finances et aux gouverneurs des banques centrales du G20, septembre.

T. Poulain-Rehm (2005), « La politique de financement des entreprises patrimoniales et familiales cotées : un test de la théorie du free cash flow », La revue du financier, $\mathrm{n}^{\circ} 153$, juillet, p. 4-20.

T. Poulain-Rehm (2006) : " Qu'est-ce qu'une entreprise familiale ? Réflexions théoriques et prescriptions empiriques », La Revue des Sciences de Gestion - Direction et gestion des entreprises, $\mathrm{n}^{\circ} 219$, mai-juin, p. 77-88.

Schulze W.S., Lubatkin M.H. et Dino R.N. (2003), « Exploring the Agency Consequences of Ownership Dispersion Among Inside Directors at Family Firms », Academy of Management Journal, vol.46, n², p. 179-194.

Schulze W.S., Lubatkin M.H. et Dino R.N. (2003), « Toward a Theory of Agency and Altruism in Family Firms », Journal of Business Venturing, vol. 18, n 4, p. 473-490.

Shleifer A. et Vishny R. (1997), «A Survey of Corporate Governance », Journal of Finance, vol. 52, p. 737-783.

Xiao J., Alhabeeb M., Hong G. et Haynes G. (2001), « Attitude Toward Risk and Risk-Taking Behavior of Business-Owning Families », Journal of Consumer Affairs, vol. 35, p. 307-325. Wacheux F. (1996), Méthodes qualitatives et recherches en gestion, Economica, Paris. 